\title{
Optimal local and remote controllers with unreliable uplink channels: An elementary proof
}

\author{
Mohammad Afshari, Student Member, IEEE, and Aditya Mahajan, Senior Member, IEEE
}

\begin{abstract}
Recently, a model of a decentralized control system with local and remote controllers connected over unreliable channels was presented in [1]. The model has a non-classical information structure that is not partially nested. Nonetheless, it is shown in [1] that the optimal control strategies are linear functions of the state estimate (which is a non-linear function of the observations). Their proof is based on a fairly sophisticated dynamic programming argument. In this note, we present an alternative and elementary proof of the result which uses common information based conditional independence and completion of squares.
\end{abstract}

Index Terms-Linear systems, certainty equivalence, separation of estimation and control, common information approach, networked control systems

\section{INTRODUCTION}

In a recent paper, a methodology for synthesizing optimal control laws for local and remote controllers for networked control of a linear system over unreliable uplink channel was presented [1]. Such models arise in applications such as temperature control in smart buildings, control of UAVs, vehicle to infrastructure communication, etc.

The model proposed in [1] is a decentralized control system with non-classical information structure. Due to the unreliable nature of the uplink channels, the information structure is not partially nested. Therefore, one cannot a priori restrict attention to linear strategies. Nonetheless, it is shown in [1] that the optimal local and remote control laws are linear functions of the state estimate (which is a non-linear function of the observations). See Theorem 1 for a precise statement of the result.

The proof technique employed in [1] uses ideas from the common information approach of [2] to compute the optimal control laws. Using a conditional independence argument, it is first shown that the local controllers can ignore the past realization of their local states without any loss of optimality [1] Lemma 1]. When attention is restricted to control strategies with such a structure the resulting information structure is partial history sharing [2]. So, in principle, the common information approach of [2] is applicable. However, there are several technical difficulties in extending the argument given in [2] for finite valued random variables to continuous random variables. The key result of [1] is to carefully resolve these technical difficulties-issues of measurability, existence of

This research was supported by NSERC under Discovery Accelerator Grant 493011.

The authors are with the Department of Electrical and Computer Engineering, McGill University, Montreal, QC, H3A0E9, Canada. Emails: mohammad.afshari2emcgill.ca, aditya.mahajan@mcgill.ca. well defined value function, and infinite dimensional strategy space - and then obtain a closed form solution of the dynamic program.

In this technical note, we provide an alternative and elementary proof of the result of [1]. Our proof also relies on the split of total information into common and local information as proposed in [2]. However, instead of using the dynamic program proposed in [2], we develop an alternative solution methodology which relies on (i) the conditional independence of the local states given the common information (which was established in [1]); and (ii) simplifying the per-step cost based on this conditional independence, orthogonality principle, and completion of squares. The key advantage of this solution approach is that it completely sidesteps the technical difficulties with measurability and existence of value functions present in a dynamic programming based approach. Given the paucity of positive results in optimal control of decentralized systems, we believe that a new solution approach is interesting in its own right.

The model considered in [1] consists of $N$ local controllers and one remote controller. For ease of exposition, we assume that $N=2$. It will be clear from the proof that the steps extend to general $N$. For the most part, we broadly follow the notation and terminology of [1], but we occasionally deviate from it to be consistent with the standard notation used in linear systems.

\section{A. Notations}

We use superscripts to indicate subsystems/controllers and subscripts to indicate time. Thus, $x_{t}^{i}$ denotes the state of subsystem $i$ at time $t$. The superscript ${ }^{\top}$ denotes transpose (of a vector or a matrix). $\mathbf{0}_{m \times n}$ is a $m \times n$ matrix with all elements being equal to zero. We omit the subscript from $\mathbf{0}_{m \times n}$ when the dimension is clear from context. Given column vectors $x$ and $y$, the notation $\operatorname{vec}(x, y)$ is a short hand for the vector formed by stacking $x$ on top of $y$. Given random variables $x$, $y$, and $z$, the notation $x \Perp y \mid z$ indicates that $x$ and $y$ are conditionally independent given $z$. Given matrices $A$ and $B$ with the same number of columns, $\operatorname{rows}(A, B)$ denotes the matrix obtained by stacking $A$ on top of $B$.

Given matrices $A, B, Q, M, R$, and $P$ of appropriate dimensions, we use the following operators:

$$
\begin{aligned}
& \mathcal{R}(P, A, B, Q, M, R)=Q+A^{\top} P A \\
& \quad-\left(M+A^{\top} P B\right)\left(R+B^{\top} P B\right)^{-1}\left(M+A^{\top} P B\right)^{\top}, \\
& \mathcal{G}(P, A, B, M, R)=-\left(R+B^{\top} P B\right)^{-1}\left(M+A^{\top} P B\right)^{\top},
\end{aligned}
$$


which denote the one step update of the discrete time Riccati equation and the gain of a linear system, respectively.

\section{Model AND Problem Formulation}

\section{A. System dynamics}

Consider a discrete-time linear dynamical system consisting of $N=2$ subsystems. $x_{t}^{i} \in \mathbb{R}^{d_{x}^{i}}$ denotes the state of subsystem $i, i \in\{1,2\}$. There is a local controller $C^{i}$ co-located with subsystem $i$. In addition, there is a remote controller $C^{0}$. The information available to the controllers will be described later. Let $u_{t}^{i} \in \mathbb{R}^{d_{u}^{i}}, i \in\{1,2\}$, denote the control action of local controller $C^{i}$ and $u_{t}^{0} \in \mathbb{R}^{d_{u}^{0}}$ denote the control action of remote controller $C^{0}$.

The initial state $x_{0}^{i}$ of subsystem $i, i \in\{1,2\}$, is random and the dynamics of subsystem $i$ is given by

$$
x_{t+1}^{i}=A^{i i} x_{t}^{i}+\left[\begin{array}{ll}
B^{i 0} & B^{i i}
\end{array}\right]\left[\begin{array}{l}
u_{t}^{0} \\
u_{t}^{i}
\end{array}\right]+w_{t}^{i},
$$

where $w_{t}^{i} \in \mathbb{R}^{d_{x}^{i}}$ is the process noise and $A^{i i}, B^{i 0}$, and $B^{i i}$ are matrices of appropriate dimensions. We assume that random variables $\left\{w_{0}^{1}, \ldots, w_{T-1}^{1}, w_{0}^{2}, \ldots, w_{T-1}^{2}\right\}$ are independent and have zero mean and finite variance. Let $x_{t}:=\operatorname{vec}\left(x_{t}^{1}, x_{t}^{2}\right)$, $u_{t}:=\operatorname{vec}\left(u_{t}^{0}, u_{t}^{1}, u_{t}^{2}\right)$, and $w_{t}:=\operatorname{vec}\left(w_{t}^{1}, w_{t}^{2}\right)$ denote the state, control actions, and noise of the overall system. Then, the system dynamics can be written as

$$
x_{t+1}=A x_{t}+B u_{t}+w_{t},
$$

where the matrices $A$ and $B$ are given by

$$
A=\left[\begin{array}{cc}
A^{11} & 0 \\
0 & A^{22}
\end{array}\right] \text { and } B=\left[\begin{array}{ccc}
B^{10} & B^{11} & 0 \\
B^{20} & 0 & B^{22}
\end{array}\right] \text {. }
$$

\section{B. Information structure}

At time $t$, the local controller $C^{i}, i \in\{1,2\}$, perfectly observes the state $x_{t}^{i}$ of subsystem $i$ and sends it to the remote controller $C^{0}$ over an unreliable packet drop channel. Let $\Gamma_{t}^{i} \in\{0,1\}$ denote the state of the channel, where $\Gamma_{t}^{i}=0$ means that the channel is in the off state where the transmitted packet gets dropped while $\Gamma_{t}^{i}=1$ means that the channel is in the on state where the transmitted packet gets delivered. Thus, $\Gamma_{t}^{i}$ is a Bernoulli random variable and we denote the packet drop probability $\mathbb{P}\left(\Gamma_{t}^{i}=0\right)$ by $p^{i}$. We use $\Gamma_{t}$ to denote $\left(\Gamma_{t}^{1}, \Gamma_{t}^{2}\right)$.

Let $z_{t}^{i}$ denote the output of the channel $i, i \in\{1,2\}$, i.e.,

$$
z_{t}^{i}=f\left(x_{t}^{i}, \Gamma_{t}^{i}\right)= \begin{cases}x_{t}^{i}, & \text { if } \Gamma_{t}^{i}=1 \\ \mathfrak{E}, & \text { if } \Gamma_{t}^{i}=0\end{cases}
$$

where $\mathfrak{E}$ denotes a dropped packet. It is assumed that there are perfect channels from $C^{0}$ to $C^{1}$ and $C^{2}$. Using these channels, $C^{0}$ can share $z_{t}:=\operatorname{vec}\left(z_{t}^{1}, z_{t}^{2}\right)$ and $u_{t-1}^{0}$ with local controllers $C^{1}$ and $C^{2}$. Note that it is possible to recover $\Gamma_{t}^{i}$ from $z_{t}^{i}$. Hence, all controllers also have access to $\Gamma_{t}$. The fact that $\Gamma_{t}$ is available at all controllers is critical to derive the main result of the model (presented in Theorem 1).
Let $H_{t}^{i}, i \in\{0,1,2\}$, denote the information available to controller $C^{i}$ to take decisions at time $t$. Then,

$$
\begin{aligned}
H_{t}^{0} & =\left\{z_{0: t}, \Gamma_{0: t}, u_{0: t-1}^{0}\right\}, \\
H_{t}^{i} & =\left\{x_{0: t}^{i}, u_{0: t-1}^{i}, z_{0: t}, \Gamma_{0: t}, u_{0: t-1}^{0}\right\}, \quad i \in\{1,2\} .
\end{aligned}
$$

Let $\mathcal{H}_{t}^{i}$ be the space of all possible realizations of $H_{t}^{i}$. Then, controller $C^{i}$ chooses it's control action according to

$$
u_{t}^{i}=g_{t}^{i}\left(H_{t}^{i}\right), \quad i \in\{0,1,2\},
$$

where the Borel measurable function $g_{t}^{i}: \mathcal{H}_{t}^{i} \rightarrow \mathbb{R}^{d_{u}^{i}}$ is called the control law of controller $C^{i}$ at time $t$. The collection $\mathbf{g}^{i}=$ $\left(g_{0}^{i}, \ldots, g_{T}^{i}\right)$ is called the control strategy of controller $C^{i}$. The collection $\mathbf{g}:=\left(\mathbf{g}^{0}, \mathbf{g}^{1}, \mathbf{g}^{2}\right)$ is called the strategy profile of the system.

\section{System performance and the optimization problem}

The system operates for a finite horizon $T$. For time $t<T$, the system incurs a per-step cost

$$
c_{t}\left(x_{t}, u_{t}\right)=\left[\begin{array}{l}
x_{t} \\
u_{t}
\end{array}\right]^{\top}\left[\begin{array}{cc}
Q_{t} & M_{t} \\
M_{t}^{\top} & R_{t}
\end{array}\right]\left[\begin{array}{l}
x_{t} \\
u_{t}
\end{array}\right]
$$

and for the terminal time $T$, the system incurs a terminal cost

$$
c_{T}\left(x_{T}\right)=x_{T}^{\top} Q_{T} x_{T},
$$

where $Q_{t}, M_{t}$, and $R_{t}$ are matrices of appropriate dimensions. We assume the following block-wise structure of $Q_{t}, M_{t}$, and $R_{t}$ :

$$
Q_{t}=\left[\begin{array}{ll}
Q_{t}^{11} & Q_{t}^{12} \\
Q_{t}^{21} & Q_{t}^{22}
\end{array}\right], \quad M_{t}=\left[\begin{array}{lll}
M_{t}^{10} & M_{t}^{11} & M_{t}^{12} \\
M_{t}^{20} & M_{t}^{21} & M_{t}^{22}
\end{array}\right],
$$

and

$$
R_{t}=\left[\begin{array}{lll}
R_{t}^{00} & R_{t}^{01} & R_{t}^{02} \\
R_{t}^{10} & R_{t}^{11} & R_{t}^{12} \\
R_{t}^{20} & R_{t}^{21} & R_{t}^{22}
\end{array}\right] .
$$

The performance of a strategy profile $\mathrm{g}$ is given by

$$
J(\mathbf{g})=\mathbb{E}^{\mathbf{g}}\left[\sum_{t=0}^{T-1} c_{t}\left(x_{t}, u_{t}\right)+c_{T}\left(x_{T}\right)\right],
$$

where the expectation is with respect to the measure induced on all the system variables by the choice of strategy profile $\mathbf{g}$.

The following assumptions are imposed on the system:

(A1) The primitive random variables $\left\{x_{0}^{1}, x_{0}^{2}, w_{0}^{1}, \ldots\right.$, $\left.w_{T-1}^{1}, w_{0}^{2}, \ldots, w_{T-1}^{2}, \Gamma_{0}^{1}, \ldots, \Gamma_{T-1}^{1}, \Gamma_{0}^{2}, \ldots, \Gamma_{T-1}^{2}\right\}$ are independent.

(A2) The variables $\left\{x_{0}^{1}, x_{0}^{2}, w_{0}^{1}, \ldots, w_{T-1}^{1}, w_{0}^{2}, \ldots, w_{T-1}^{2}\right\}$ have zero mean and finite variance. We use $\Sigma_{t}^{i}$ and $\Sigma_{x}^{i}$ to denote the variance of $w_{t}^{i}$ and $x_{0}^{i}$ respectively.

(A3) For each $t$, the matrix $\left[\begin{array}{cc}Q_{t} & M_{t} \\ M_{t}^{\top} & R_{t}\end{array}\right]$ is symmetric and positive semi-definite, and the matrix $R_{t}$ is symmetric and positive definite.

We are interested in the following optimization problem.

Problem 1 In the model described above, find a strategy profile $\mathbf{g}^{*}=\left(\mathbf{g}^{*, 0}, \mathbf{g}^{*, 1}, \mathbf{g}^{*, 2}\right)$ that minimizes (6), i.e.,

$$
J^{*}:=J\left(\mathbf{g}^{*}\right)=\inf _{\mathbf{g}} J(\mathbf{g}),
$$

where the infimum is taken over all strategy profiles of the form (5). 


\section{Some remarks}

The per-step cost function defined above differs slightly from the per-step cost function considered in [1] in the following ways:

- In [1], the matrix $\left[\begin{array}{cc}Q_{t} & M_{t} \\ M_{t}^{\top} & R_{t}\end{array}\right]$ was denoted by $R_{t}$. We follow the standard notation here.

- In [1], it was assumed that the performance of a strategy profile is

$$
\mathbb{E}^{\mathbf{g}}\left[\sum_{t=0}^{T} c_{t}\left(x_{t}, u_{t}\right)\right] .
$$

This is effectively the same as assuming that there is no terminal cost (i.e., $Q_{T+1}=0$ ) and therefore the terminal control actions $u_{T}^{i}$ are 0 for both local and remote controllers. To avoid such triviality, we assume a performance function of the form (6).

\section{MAIN RESULT}

In this section, we restate the main results of [1] but we present them in a slightly different manner.

\section{A. Common information based estimates}

Following [2], we define the common information $H_{t}^{\mathrm{com}}$ between agents as

$$
H_{t}^{\mathrm{com}}=H_{t}^{0} \cap H_{t}^{1} \cap H_{t}^{2} .
$$

The information structure of the model (4) implies that $H_{t}^{\mathrm{com}}=H_{t}^{0}=\left\{z_{0: t}, \Gamma_{0: t}, u_{0: t-1}^{0}\right\}$.

Now we define the common information based "estimates" of the state and control actions and the corresponding "estimation errors" as follows:

$$
\begin{array}{ll}
\hat{x}_{t}=\mathbb{E}\left[x_{t} \mid H_{t}^{\mathrm{com}}\right], & \tilde{x}_{t}=x_{t}-\hat{x}_{t}, \\
\hat{u}_{t}=\mathbb{E}\left[u_{t} \mid H_{t}^{\mathrm{com}}\right], & \tilde{u}_{t}=u_{t}-\hat{u}_{t} .
\end{array}
$$

For ease of notation, we use $\hat{x}_{t}^{i}$ to denote the $i_{t h}$ component of $\hat{x}_{t}$, i.e., $\hat{x}_{t}=\operatorname{vec}\left(\hat{x}_{t}^{1}, \hat{x}_{t}^{2}\right)$. Similar interpretation holds for $\tilde{x}_{t}^{i}, \hat{u}_{t}^{i}$, and $\tilde{u}_{t}^{i}$.

It can be shown that the state estimates and the estimation error satisfy the following property.

Lemma 1 The state estimates and estimation errors evolve as follows: for $i \in\{1,2\}$,

$$
\hat{x}_{0}^{i}= \begin{cases}0, & \text { if } \Gamma_{0}^{i}=0 \\ x_{0}^{i}, & \text { if } \Gamma_{0}^{i}=1\end{cases}
$$

and for $t>0$,

$$
\hat{x}_{t+1}^{i}= \begin{cases}A^{i i} \hat{x}_{t}^{i}+B^{i 0} u_{t}^{0}+B^{i i} \hat{u}_{t}^{i}, & \text { if } \Gamma_{t+1}^{i}=0 \\ x_{t+1}^{i}, & \text { if } \Gamma_{t+1}^{i}=1 .\end{cases}
$$

Therefore,

$$
\tilde{x}_{0}^{i}= \begin{cases}x_{0}^{i}, & \text { if } \Gamma_{0}^{i}=0 \\ 0, & \text { if } \Gamma_{0}^{i}=1\end{cases}
$$

and for $t>0$,

$$
\tilde{x}_{t+1}^{i}= \begin{cases}A^{i i} \tilde{x}_{t}^{i}+B^{i i} \tilde{u}_{t}^{i}+w_{t}^{i}, & \text { if } \Gamma_{t+1}^{i}=0 \\ 0, & \text { if } \Gamma_{t+1}^{i}=1 .\end{cases}
$$

A proof is presented in Sec. IV-D

Remark 1 Lemma 1 along with the definition of the state and control estimates (7) and (8) and the information structure (4) imply that all controllers know the value of $\operatorname{vec}\left(\hat{x}_{t}^{1}, \hat{x}_{t}^{2}\right)$ at time $t$. An immediate consequence of this is that controller $C^{i}$ knows the value of $\tilde{x}_{t}^{i}$ at time $t$. The main result of the model, explained in the next section, is that the optimal control action at controller $C^{i}$ is linear in $\left(\hat{x}_{t}, \tilde{x}_{t}^{i}\right)$.

\section{B. Structure of optimal control laws}

In order to present the main result of [1], we recursively define matrices $\left\{P_{t}\right\}_{t=1}^{T}$ as follows: $P_{T}=Q_{T}$ and for $t \in$ $\{T-1, \ldots, 1\}$,

$$
P_{t}=\mathcal{R}\left(P_{t+1}, A, B, Q_{t}, M_{t}, R_{t}\right) .
$$

Furthermore, let $P_{t}^{i i}$ denote the $(i, i)$-th block of $P_{t}$. Then for $i \in\{1,2\}$, recursively define the matrices $\left\{\Pi_{t}^{i}\right\}_{t=1}^{T}$ and $\left\{\tilde{P}_{t}^{i}\right\}_{t=1}^{T}$ as follows: $\Pi_{T}^{i}=Q_{T}^{i i}$ and $\tilde{P}_{T}^{i}=Q_{T}^{i i}$ and for $t \in$ $\{T-1, \ldots, 1\}$, let

$$
\tilde{P}_{t}^{i}=\mathcal{R}\left(\Pi_{t+1}^{i}, A^{i i}, B^{i i}, Q_{t}^{i i}, M_{t}^{i i}, R_{t}^{i i}\right) .
$$

and

$$
\Pi_{t+1}^{i}=\left(1-p^{i}\right) P_{t+1}^{i i}+p^{i} \tilde{P}_{t+1}^{i} .
$$

The main result of [1] is the following.

Theorem 1 The optimal control strategy for Problem $\square$ is given by

$$
\left[\begin{array}{c}
u_{t}^{0} \\
\hat{u}_{t}^{1} \\
\hat{u}_{t}^{2}
\end{array}\right]=-K_{t} \hat{x}_{t}
$$

and

$$
\tilde{u}_{t}^{i}=-\tilde{K}_{t}^{i} \tilde{x}_{t}^{i}, \quad i \in\{1,2\},
$$

where the time evolution of $\hat{x}_{t}$ and $\tilde{x}_{t}$ are given by Lemma 1 . The gains $\left\{K_{t}\right\}_{t=0}^{T-1}$ and $\left\{\tilde{K}_{t}\right\}_{t=0}^{T-1}$ are given by

$$
\begin{aligned}
K_{t} & =\mathcal{G}\left(P_{t+1}, A, B, M_{t}, R_{t}\right), \\
\tilde{K}_{t}^{i} & =\mathcal{G}\left(\Pi_{t+1}^{i}, A^{i i}, B^{i i}, M_{t}^{i i}, R_{t}^{i i}\right), \quad i \in\{1,2\},
\end{aligned}
$$

where the matrices $\left\{P_{t}\right\}_{t=1}^{T},\left\{\Pi_{t}^{i}\right\}_{t=1}^{T}$, and $\left\{\tilde{P}^{i}\right\}_{t=1}^{T}$ are given by (9), (10), and (11).

Remark 2 Let $K_{t}=\operatorname{rows}\left(K_{t}^{0}, K_{t}^{1}, K_{t}^{2}\right)$. Then, Theorem 1 implies that the optimal control actions are given by

$$
\begin{aligned}
& u_{t}^{0}=-K_{t}^{0} \hat{x}_{t}, \\
& u_{t}^{i}=-K_{t}^{i} \hat{x}_{t}+\tilde{K}_{t}^{i}\left(x_{t}^{i}-\hat{x}_{t}^{i}\right), \quad i \in\{1,2\} .
\end{aligned}
$$

Such a control law is feasible because, $\tilde{x}_{t}^{i}$ is available at controller $C^{i}$ as explained in Remark 1.

The structure of the control laws (14)-(15) implies that the optimal action is a linear function of the state estimate $\hat{x}_{t}$. Note that the evolution of the state estimate, given by Lemma 1, is a non-linear function of the data available at controller $C^{i}$, $i \in\{1,2\}$.

Remark 3 Note that the result does not depend on the distribution of the noise processes $\left\{w_{t}^{i}\right\}_{t \geq 0}, i \in\{1,2\}$, as long 
as the random variables $\left\{w_{0}^{1}, \ldots, w_{T-1}^{1}, w_{0}^{2}, \ldots, w_{T-1}^{2}\right\}$ are independent and have finite second moment. For convenience we have presented the result under the additional assumption that the noise is zero-mean but that assumption can be relaxed using a simple change of variables.

\section{Proof of the Main Result}

\section{A. Roadmap of the proof}

Our proof is based on the following fact which is typically referred to as completion of squares in the literature.

Fact Given a linear system $x_{t+1}=A x_{t}+B u_{t}+w_{t}$, the quadratic cost

$$
\sum_{t=0}^{T-1}\left[x_{t}^{\top} Q_{t} x_{t}+u_{t}^{\top} R_{t} u_{t}\right]+x_{T}^{\top} Q_{T} x_{T}
$$

may be rewritten as

$x_{0}^{\top} P_{0} x_{0}+\sum_{t=0}^{T-1}\left(u_{t}+L_{t} x_{t}\right)^{\top} \Delta_{t}\left(u_{t}+L_{t} x_{t}\right)+\sum_{t=0}^{T-1} w_{t}^{\top} P_{t+1} w_{t}$,

where $P_{T}=Q_{T}$ and for $t \in\{T-1, \ldots, 0\}, P_{t}=$ $\mathcal{R}\left(P_{t+1}, A, B, Q_{t}, \mathbf{0}, R_{t}\right), \quad L_{t}=\mathcal{G}\left(P_{t+1}, A, B, \mathbf{0}, R_{t}\right)$, and $\Delta_{t}=\left(R_{t}+B^{\top} P_{t+1} B\right)$.

Using this fact, one can prove the structure of optimal strategy for the centralized control of stochastic linear systems for both complete and partial state observation. See, for example, [3, Chapter 8]. However, the completion of squares argument does not work directly for decentralized control systems.

In our proof, we exploit a fundamental property of the model, which was established in [1, Claim 2] and is formally stated as Lemma 2 below: $x_{t}^{1} \Perp x_{t}^{2} \mid H_{t}^{\text {com }}$. As a consequence of this conditional independence, the past realizations $\left(x_{0: t-1}^{i}, u_{0: t-1}^{i}\right)$ are irrelevant at controller $C^{i}$ and may be shed without loss of optimality. This follows from Blackwell's principle of irrelevant information [4] as generalized to decentralized control systems in [5]. The simplified structure of the optimal controller was established in [1, Lemma 1] and is formally stated as Lemma 3 below.

Using these two results and basic properties of conditional expectations, we prove the structure of the dynamics of the state estimates and the estimation error (Lemma 1). This structure was also established in [1, Theorem 3] as part of the result that establishes the structure of the optimal controller However, as we show below, one only needs the conditional independence property of Lemma 2 and its consequences to establish Lemma 1

As a next step, we use orthogonal projection and the specific form of the information structure to simplify the per-step cost (Lemma 5). We combine this simplified form of the cost with the dynamics of the state estimates and estimation error (established in Lemma 1) to prove completion of squares result for the cost (Theorem 2) tailored to the specific model of the system.

Subsequently, we follow the standard steps of the "completion of squares" argument to establish the structure of the optimal strategy.
B. Conditional independence of local states and its implications

A key property of the model established in [1, Claim 2] is the following.

Lemma 2 For any control strategy profile $\mathrm{g}$ of the form (5),

$$
x_{t}^{1} \Perp x_{t}^{2} \mid H_{t}^{\text {com }} .
$$

Furthermore, it is shown in [1, Lemma 1] that the above conditional independence implies the following.

Lemma 3 In Problem $\square$ there is no loss of optimality to restrict attention to local controllers of the form

$$
u_{t}^{i}=g_{t}^{i}\left(x_{t}^{i}, H_{t}^{\mathrm{com}}\right), \quad i \in\{1,2\} .
$$

An immediate consequence of the above lemma is the following.

Corollary 1 For any control strategy profile $\mathrm{g}$ of the form (16), we have the following:

1) $\left(x_{t}^{1}, u_{t}^{1}\right) \Perp\left(x_{t}^{2}, u_{t}^{2}\right) \mid H_{t}^{\text {com }}$.

2) $\left(\tilde{x}_{t}^{1}, \tilde{u}_{t}^{1}\right) \Perp\left(\tilde{x}_{t}^{2}, \tilde{u}_{t}^{2}\right) \mid H_{t}^{\text {com }}$.

Proof Property 1 follows from the Lemma 2 and the structure of the control strategy. Property 2 follows from Property 1, Eqs. (7) and (8) and the fact that $\hat{x}_{t}^{i}$ and $\hat{u}_{t}^{i}$ are functions of $H_{t}^{\text {com }}$.

\section{Some preliminary properties}

Lemma 4 For any control strategy profile $\mathrm{g}$ of the form (5), we have the following:

(H1) $\hat{u}_{t}^{0}=u_{t}^{0}$ and $\tilde{u}_{t}^{0}=\mathbf{0}$. Thus, $\hat{u}_{t}=\operatorname{vec}\left(u_{t}^{0}, \hat{u}_{t}^{1}, \hat{u}_{t}^{2}\right)$ and $\tilde{u}_{t}=\operatorname{vec}\left(\mathbf{0}, \tilde{u}_{t}^{1}, \tilde{u}_{t}^{2}\right)$.

(H2) $\mathbb{E}\left[\tilde{x}_{t} \mid H_{t}^{\text {com }}\right]=0$ and $\mathbb{E}\left[\tilde{u}_{t} \mid H_{t}^{\text {com }}\right]=0$.

(H3) For any matrix $W$ of appropriate dimensions, $\mathbb{E}\left[\hat{s}_{t}^{\top} W \tilde{s}_{t}\right]=0$, where $\hat{s}_{t}=\operatorname{vec}\left(\hat{x}_{t}, \hat{u}_{t}\right)$ and $\tilde{s}_{t}=$ $\operatorname{vec}\left(\tilde{x}_{t}, \tilde{u}_{t}\right)$.

Furthermore, if the strategy profile is of the form (16), we have:

(H4) For any matrix $W$ of appropriate dimensions, $\mathbb{E}\left[\left(\tilde{s}_{t}^{1}\right)^{\top} W \tilde{s}_{t}^{2}\right]=0$, where $\tilde{s}_{t}^{i}=\operatorname{vec}\left(\tilde{x}_{t}^{i}, \tilde{u}_{t}^{i}\right)$.

Proof Property (H1) follows from the fact that $u_{t}^{0}$ is a measurable function of $H_{t}^{0}$ (which is the same as $H_{t}^{\text {com }}$ ).

Property (H2) is a standard property of error estimates and can be shown as follows:

$$
\mathbb{E}\left[\tilde{x}_{t} \mid H_{t}^{\mathrm{com}}\right]=\mathbb{E}\left[x_{t}-\mathbb{E}\left[x_{t} \mid H_{t}^{\mathrm{com}}\right] \mid H_{t}^{\mathrm{com}}\right]=0 .
$$

Property (H3) follows from the generalized orthogonal principle and can be shown as follows:

$$
\mathbb{E}\left[\hat{s}_{t}^{\top} W \tilde{s}_{t}\right]=\mathbb{E}\left[\mathbb{E}\left[\hat{s}_{t}^{\top} W \tilde{s}_{t} \mid H_{t}^{\text {com }}\right]\right]=\mathbb{E}[\hat{s}_{t}^{\top} W \underbrace{\mathbb{E}\left[\tilde{s}_{t} \mid H_{t}^{\text {com }}\right]}_{=0 \text { (by (H2)) }}] .
$$

Property (H4) follows from Corollary 11 and (H2). 


\section{Proof of Lemma 1}

By definition, $H_{t+1}^{\text {com }}=H_{t}^{\text {com }} \cup\left\{z_{t+1}^{1}, z_{t+1}^{2}, \Gamma_{t+1}^{1}, \Gamma_{t+1}^{2}, u_{t}^{0}\right\}$. Thus,

$$
\begin{aligned}
\hat{x}_{t+1}^{i} & =\mathbb{E}\left[x_{t+1}^{i} \mid H_{t+1}^{\mathrm{com}}\right] \\
& =\mathbb{E}\left[x_{t+1}^{i} \mid H_{t}^{\mathrm{com}}, z_{t+1}^{1}, z_{t+1}^{2}, \Gamma_{t+1}^{1}, \Gamma_{t+1}^{2}, u_{t}^{0}\right] \\
& =\mathbb{E}\left[x_{t+1}^{i} \mid H_{t}^{\mathrm{com}}, z_{t+1}^{i}, \Gamma_{t+1}^{i}\right]
\end{aligned}
$$

where we can remove $z_{t+1}^{-i}$ (where $-i$ means the controller other than $i), \Gamma_{t+1}^{-i}$, and $u_{t}^{0}$ due to the following reasons:

- By (3), $z_{t+1}^{-i}=f\left(x_{t+1}^{-i}, \Gamma_{t+1}^{-i}\right)$ and hence conditionally independent of $x_{t+1}^{i}$ given $H_{t}^{\text {com }}$ due to (A1), Lemma 2 and Lemma 3.

- $\Gamma_{t+1}^{-i}$ is conditionally independent of $x_{t+1}^{i}$ given $\left\{H_{t}^{\mathrm{com}}, u_{t}^{0}\right\}$ due to (1) and (A1).

- $u_{t}^{0}=g_{t}^{0}\left(H_{t}^{0}\right)$ and hence may be removed from the conditioning ( since $H_{t}^{\text {com }}=H_{t}^{0}$ ).

Now, we consider the two cases $\Gamma_{t+1}^{i}=0$ and $\Gamma_{t+1}^{i}=1$ separately. When $\Gamma_{t+1}^{i}=0, z_{t+1}^{i}=\mathfrak{E}$ and from 17) we have

$$
\begin{aligned}
\hat{x}_{t+1}^{i} & =\mathbb{E}\left[x_{t+1}^{i} \mid H_{t}^{\mathrm{com}}, z_{t+1}^{i}=\mathfrak{E}, \Gamma_{t+1}^{i}=0\right] \\
& \stackrel{(a)}{=} \mathbb{E}\left[x_{t+1}^{i} \mid H_{t}^{\mathrm{com}}\right] \\
& \stackrel{(b)}{=} A^{i i} \hat{x}_{t}^{i}+B^{i 0} u_{t}^{0}+B^{i i} \hat{u}_{t}^{i}
\end{aligned}
$$

where $(a)$ follows from (A1) and (b) follows from (1), (7), (8), (H1), (A1), and (A2). Consequently,

$$
\tilde{x}_{t+1}^{i}=x_{t+1}^{i}-\hat{x}_{t+1}^{i}=A^{i i} \tilde{x}_{t}^{i}+B^{i i} \tilde{u}_{t}^{i}+w_{t}^{i} .
$$

Now consider the case when $\Gamma_{t+1}^{i}=1$, i.e., $z_{t+1}^{i}=x_{t+1}^{i}$. Therefore,

$$
\hat{x}_{t+1}^{i}=\mathbb{E}\left[x_{t+1}^{i} \mid H_{t}^{\mathrm{com}}, z_{t+1}^{i}=x_{t+1}^{i}, \Gamma_{t+1}^{i}=1\right]=x_{t+1}^{i} .
$$

Consequently, $\tilde{x}_{t+1}^{i}=x_{t+1}^{i}-\hat{x}_{t+1}^{i}=0$.

\section{E. Orthogonal projection for per-step cost}

Lemma 5 For any strategy profile of the form (16), we have

$$
\begin{aligned}
& \mathbb{E}\left[x_{t}^{\top} Q_{t} x_{t}\right]=\mathbb{E}\left[\hat{x}_{t}^{\top} Q_{t} \hat{x}_{t}+\sum_{i \in\{1,2\}}\left(\tilde{x}_{t}^{i}\right)^{\top} Q_{t}^{i i} \tilde{x}_{t}^{i}\right], \\
& \mathbb{E}\left[u_{t}^{\top} R_{t} u_{t}\right]=\mathbb{E}\left[\hat{u}_{t}^{\top} R_{t} \hat{u}_{t}+\sum_{i \in\{1,2\}}\left(\tilde{u}_{t}^{i}\right)^{\top} R_{t}^{i i} \tilde{u}_{t}^{i}\right], \\
& \mathbb{E}\left[x_{t}^{\top} M_{t} u_{t}\right]=\mathbb{E}\left[\hat{x}_{t}^{\top} M_{t} \hat{u}_{t}+\sum_{i \in\{1,2\}}\left(\tilde{x}_{t}^{i}\right)^{\top} M_{t}^{i i} \tilde{u}_{t}^{i}\right] .
\end{aligned}
$$

Thus, we have that

$$
\begin{aligned}
& \mathbb{E}\left[\left[\begin{array}{l}
x_{t} \\
u_{t}
\end{array}\right]^{\top}\left[\begin{array}{cc}
Q_{t} & M_{t} \\
M_{t}^{\top} & R_{t}
\end{array}\right]\left[\begin{array}{l}
x_{t} \\
u_{t}
\end{array}\right]\right]=\mathbb{E}\left[\left[\begin{array}{l}
\hat{x}_{t} \\
\hat{u}_{t}
\end{array}\right]^{\top}\left[\begin{array}{cc}
Q_{t} & M_{t} \\
M_{t}^{\top} & R_{t}
\end{array}\right]\left[\begin{array}{l}
\hat{x}_{t} \\
\hat{u}_{t}
\end{array}\right]\right] \\
& +\sum_{i \in\{1,2\}} \mathbb{E}\left[\left[\begin{array}{c}
\tilde{x}_{t}^{i} \\
\tilde{u}_{t}^{i}
\end{array}\right]^{\top}\left[\begin{array}{cc}
Q_{t}^{i i} & M_{t}^{i i} \\
\left(M_{t}^{i i}\right)^{\top} & R_{t}^{i i}
\end{array}\right]\left[\begin{array}{c}
\tilde{x}_{t}^{i} \\
\tilde{u}_{t}^{i}
\end{array}\right]\right]
\end{aligned}
$$

PROOF To show (18), we recall that $x_{t}=\hat{x}_{t}+\tilde{x}_{t}$. Thus,

$$
\mathbb{E}\left[x_{t}^{\top} Q_{t} x_{t}\right]=\mathbb{E}\left[\hat{x}_{t}^{\top} Q_{t} \hat{x}_{t}+\tilde{x}_{t}^{\top} Q_{t} \tilde{x}_{t}+2 \hat{x}_{t}^{\top} Q_{t} \tilde{x}_{t}\right] .
$$

Consider the second term of (21)

$$
\mathbb{E}\left[\tilde{x}_{t}^{\top} Q_{t} \tilde{x}_{t}\right]=\sum_{i \in\{1,2\}} \mathbb{E}\left[\left(\tilde{x}_{t}^{i}\right)^{\top} Q_{t}^{i i} \tilde{x}_{t}^{i}\right]+2 \underbrace{\mathbb{E}\left[\left(\tilde{x}_{t}^{1}\right)^{\top} Q_{t}^{12} \tilde{x}_{t}^{2}\right]}_{=0 \text { (by (H4)) }} .
$$

Substituting (22) in (21) and observing that the third term of (21) is 0 due to (H3), we get (18).

Eqs. (19) and 20 can be proved in a similar manner.

\section{F. A change of variables}

For ease of notation, we define

$$
\begin{aligned}
& \hat{x}_{t+1}^{i, \mathrm{OFF}}=A^{i i} \hat{x}_{t}^{i}+B^{i 0} u_{t}^{0}+B^{i i} \hat{u}_{t}^{i}, \\
& \tilde{x}_{t+1}^{i, \mathrm{OFF}}=A^{i i} \tilde{x}_{t}^{i}+B^{i i} \tilde{u}_{t}^{i}+w_{t}^{i} .
\end{aligned}
$$

Thus, we can write

$$
\hat{x}_{t+1}^{i}= \begin{cases}\hat{x}_{t+1}^{i, \mathrm{OFF}}, & \text { if } \Gamma_{t+1}^{i}=0 \\ x_{t+1}^{i}, & \text { if } \Gamma_{t+1}^{i}=1,\end{cases}
$$

and

$$
\tilde{x}_{t+1}^{i}= \begin{cases}\tilde{x}_{t+1}^{i, \mathrm{OFF}}, & \text { if } \Gamma_{t+1}^{i}=0 \\ 0, & \text { if } \Gamma_{t+1}^{i}=1 .\end{cases}
$$

Let $\hat{x}_{t}^{\mathrm{OFF}}=\operatorname{vec}\left(\hat{x}_{t}^{1, \mathrm{OFF}}, \hat{x}_{t}^{2, \mathrm{OFF}}\right)$ and $\tilde{x}_{t}^{\mathrm{OFF}}=\operatorname{vec}\left(\tilde{x}_{t}^{1, \mathrm{OFF}}, \tilde{x}_{t}^{2, \mathrm{OFF}}\right)$. It follows that $\hat{x}_{t+1}^{i, \text { OFF }}+\tilde{x}_{t+1}^{i, \text { OFF }}=x_{t+1}^{i}$ and

$$
\hat{x}_{t+1}^{\mathrm{OFF}}=A \hat{x}_{t}+B \hat{u}_{t} \text {. }
$$

Lemma 6 For any strategy profile of the form (5), we have the following:

(H5) For any matrix $W$ of appropriate dimensions, $\mathrm{E}\left[\hat{x}_{t}^{\mathrm{OFF}} W \tilde{x}_{t}^{\mathrm{OFF}}\right]=0$.

Furthermore, if the strategy profile is of the form (16), we have:

(H6) For any matrix $W$ of appropriate dimensions, $\mathbb{E}\left[\left(\tilde{x}_{t}^{1, \mathrm{OFF}}\right)^{\top} W \tilde{x}_{t}^{2, \mathrm{OFF}}\right]=0$.

ProOF Property (H5) follows immediately from (H3). Property (H6) follows immediately from (H4) and (A1).

Lemma 7 For any strategy profile of the form (16), we have the following:

$$
\begin{aligned}
& \mathbb{E}\left[\hat{x}_{t+1}^{\top} P_{t+1} \hat{x}_{t+1}+\sum_{i \in\{1,2\}}\left(\tilde{x}_{t+1}^{i}\right)^{\top} \tilde{P}_{t+1}^{i} \tilde{x}_{t+1}^{i}\right] \\
& \quad=\mathbb{E}\left[\left(\hat{x}_{t+1}^{\mathrm{OFF}}\right)^{\top} P_{t+1} \hat{x}_{t+1}^{\mathrm{OFF}}+\sum_{i \in\{1,2\}}\left(\tilde{x}_{t+1}^{i, \mathrm{OFF}}\right)^{\top} \Pi_{t+1}^{i} \tilde{x}_{t+1}^{i, \mathrm{OFF}}\right] .
\end{aligned}
$$

Proof We compute the conditional value of the left hand side given the realization of $\Gamma_{t+1}=\left(\Gamma_{t+1}^{1}, \Gamma_{t+1}^{2}\right)$ and using Lemma 1 We have four cases

1) $\Gamma_{t+1}=(0,0)$ : In this case $\hat{x}_{t+1}=\hat{x}_{t+1}^{\mathrm{OFF}}$ and $\tilde{x}_{t+1}=$ $\tilde{x}_{t+1}^{\text {OFF }}$. Thus,

$$
\begin{gathered}
\mathbb{E}\left[\hat{x}_{t+1}^{\top} P_{t+1} \hat{x}_{t+1}+\sum_{i \in\{1,2\}}\left(\tilde{x}_{t+1}^{i}\right)^{\top} \tilde{P}_{t+1}^{i} \tilde{x}_{t+1}^{i} \mid \Gamma_{t+1}=(0,0)\right] \\
=\mathbb{E}\left[\left(\hat{x}_{t+1}^{\mathrm{OFF}}\right)^{\top} P_{t+1} \hat{x}_{t+1}^{\mathrm{OFF}}+\sum_{i \in\{1,2\}}\left(\tilde{x}_{t+1}^{i, \mathrm{OFF}}\right)^{\top} \tilde{P}_{t+1}^{i} \tilde{x}_{t+1}^{i, \mathrm{OFF}}\right] .
\end{gathered}
$$


2) $\Gamma_{t+1}=(1,0)$ : In this case $\hat{x}_{t+1}=\operatorname{vec}\left(x_{t+1}^{1}, \hat{x}_{t+1}^{2, \text { OFF }}\right)=$ $\hat{x}_{t+1}^{\text {OFF }}+\operatorname{vec}\left(\tilde{x}_{t+1}^{1, \text { OFF }}, \mathbf{0}\right)$ and $\tilde{x}_{t+1}=\operatorname{vec}\left(\mathbf{0}, \tilde{x}_{t+1}^{2, \text { OFF }}\right)$. Thus,

$$
\begin{gathered}
\mathbb{E}\left[\hat{x}_{t+1}^{\top} P_{t+1} \hat{x}_{t+1}+\sum_{i \in\{1,2\}}\left(\tilde{x}_{t+1}^{i}\right)^{\top} \tilde{P}_{t+1}^{i} \tilde{x}_{t+1}^{i} \mid \Gamma_{t+1}=(1,0)\right] \\
=\mathbb{E}\left[\left(\hat{x}_{t+1}^{\mathrm{OFF}}\right)^{\top} P_{t+1} \hat{x}_{t+1}^{\mathrm{OFF}}+\left(\tilde{x}_{t+1}^{1, \mathrm{OFF}}\right)^{\top} P_{t+1}^{11} \tilde{x}_{t+1}^{1, \mathrm{OFF}}\right. \\
\left.+\left(\tilde{x}_{t+1}^{2, \mathrm{OFF}}\right)^{\top} \tilde{P}_{t+1}^{2} \tilde{x}_{t+1}^{2, \mathrm{OFF}}\right] .
\end{gathered}
$$

3) $\Gamma_{t+1}=(0,1)$ : Similar to case 2$)$, we can show that

$$
\begin{gathered}
\mathbb{E}\left[\hat{x}_{t+1}^{\top} P_{t+1} \hat{x}_{t+1}+\sum_{i \in\{1,2\}}\left(\tilde{x}_{t+1}^{i}\right)^{\top} \tilde{P}_{t+1}^{i} \tilde{x}_{t+1}^{i} \mid \Gamma_{t+1}=(0,1)\right] \\
=\mathbb{E}\left[\left(\hat{x}_{t+1}^{\mathrm{OFF}}\right)^{\top} P_{t+1} \hat{x}_{t+1}^{\mathrm{OFF}}+\left(\tilde{x}_{t+1}^{1, \mathrm{OFF}}\right)^{\top} \tilde{P}_{t+1}^{1} \tilde{x}_{t+1}^{1, \mathrm{OFF}}\right. \\
\left.+\left(\tilde{x}_{t+1}^{2, \mathrm{OFF}}\right)^{\top} P_{t+1}^{22} \tilde{x}_{t+1}^{2, \mathrm{OFF}}\right] .
\end{gathered}
$$

4) $\Gamma_{t+1}=(1,1)$ : In this case, $\hat{x}_{t+1}=x_{t+1}=\hat{x}_{t+1}^{\mathrm{OFF}}+\tilde{x}_{t+1}^{\mathrm{OFF}}$ and $\tilde{x}_{t+1}=\mathbf{0}$. Thus,

$$
\begin{gathered}
\mathbb{E}\left[\hat{x}_{t+1}^{\top} P_{t+1} \hat{x}_{t+1}+\sum_{i \in\{1,2\}}\left(\tilde{x}_{t+1}^{i}\right)^{\top} \tilde{P}_{t+1}^{i} \tilde{x}_{t+1}^{i} \mid \Gamma_{t+1}=(1,1)\right] \\
=\mathbb{E}\left[\left(\hat{x}_{t+1}^{\mathrm{OFF}}\right)^{\top} P_{t+1} \hat{x}_{t+1}^{\mathrm{OFF}}+\left(\tilde{x}_{t+1}^{\mathrm{OFF}}\right)^{\top} P_{t+1} \tilde{x}_{t+1}^{\mathrm{OFF}}\right. \\
\left.+2\left(\hat{x}_{t+1}^{\mathrm{OFF}}\right)^{\top} P_{t+1} \tilde{x}_{t+1}^{\mathrm{OFF}}\right] \\
=\mathbb{E}\left[\left(\hat{x}_{t+1}^{\mathrm{OFF}}\right)^{\top} P_{t+1} \hat{x}_{t+1}^{\mathrm{OFF}}+\sum_{i \in\{1,2\}}\left(\tilde{x}_{t+1}^{i, \mathrm{OFF}}\right)^{\top} P_{t+1}^{i i} \tilde{x}_{t+1}^{i, \mathrm{OFF}}\right]
\end{gathered}
$$

where the last equality follows from (H5) and (H6).

Combining these four cases and using the law of total probability, we get (26).

\section{G. Completion of squares}

Lemma 8 Let $x \in \mathbb{R}^{d_{x}}, u \in \mathbb{R}^{d_{u}}$, and $w \in \mathbb{R}^{d_{x}}$ be random variables defined on a common probability space. Suppose $w$ is zero mean with finite covariance and independent of $(x, u)$. Let $x_{+}=A x+B u+w$, where $A$ and $B$ are matrices of appropriate dimensions. Then given matrices $P, Q, M$, and $R$ of appropriate dimensions,

$$
\begin{aligned}
\mathbb{E}\left[\left[\begin{array}{l}
x \\
u
\end{array}\right]^{\top}\left[\begin{array}{cc}
Q & M \\
M^{\top} & R
\end{array}\right]\left[\begin{array}{l}
x \\
u
\end{array}\right]+x_{+}^{\top} P x_{+}\right] \\
\quad=\mathbb{E}\left[x^{\top} P_{+} x+(u+K x)^{\top} \Delta(u+K x)+w^{\top} P w\right],
\end{aligned}
$$

where

$$
\begin{aligned}
\Delta & =R+B^{\top} P B, \\
K & =\Delta^{-1}\left[M^{\top}+B^{\top} P A\right], \\
P_{+} & =Q+A^{\top} P A-K^{\top} \Delta K .
\end{aligned}
$$

Proof Since $w$ is zero mean and independent of $(x, u)$, we have

$$
\mathbb{E}\left[x_{+}^{\top} P x_{+}\right]=\mathbb{E}\left[(A x+B u)^{\top} P(A x+B u)+w^{\top} P w\right]
$$

The result follows by expanding both sides and comparing coefficients.

By combining Lemmas 5, 7 and 8 we get the following.
Lemma 9 For any strategy profile of the form (16),

$$
\begin{gathered}
\mathbb{E}\left[c_{t}\left(x_{t}, u_{t}\right)+\hat{x}_{t+1}^{\top} P_{t+1} \hat{x}_{t+1}+\sum_{i \in\{1,2\}}\left(\tilde{x}_{t+1}^{i}\right)^{\top} \tilde{P}_{t+1}^{i} \tilde{x}_{t+1}^{i}\right] \\
=\mathbb{E}\left[\hat{x}_{t}^{\top} P_{t} \hat{x}_{t}+\left(\hat{u}_{t}+K_{t} \hat{x}_{t}\right)^{\top} \Delta_{t}\left(\hat{u}_{t}+K_{t} \hat{x}_{t}\right)\right. \\
\quad+\sum_{i \in\{1,2\}}\left[\left(\tilde{x}_{t}^{i}\right)^{\top} \tilde{P}_{t}^{i} \tilde{x}_{t}^{i}+\left(\tilde{u}_{t}+\tilde{K}_{t} \tilde{x}_{t}\right)^{\top} \tilde{\Delta}_{t}\left(\tilde{u}_{t}+\tilde{K}_{t} \tilde{x}_{t}\right)\right. \\
\left.\left.+\left(w_{t}^{i}\right)^{\top} \tilde{\Pi}_{t+1}^{i} w_{t}^{i}\right]\right] .
\end{gathered}
$$

Theorem 2 For any strategy profile $\mathrm{g}$ of the form (16),

$$
\begin{aligned}
J(\mathbf{g})=\mathbb{E}^{\mathbf{g}}[ & \hat{x}_{0}^{\top} P_{t} \hat{x}_{0}+\sum_{i \in\{1,2\}}\left(\tilde{x}_{0}^{i}\right)^{\top} \tilde{P}_{0}^{i} \tilde{x}_{0}^{i} \\
& +\sum_{s=0}^{T-1}\left(\hat{u}_{s}+K_{s} \hat{x}_{s}\right)^{\top} \Delta_{s}\left(\hat{u}_{s}+K_{s} \hat{x}_{s}\right) \\
& +\sum_{s=0}^{T-1} \sum_{i \in\{1,2\}}\left(\tilde{u}_{s}^{i}+\tilde{K}_{s}^{i} \tilde{x}_{s}^{i}\right)^{\boldsymbol{\top}} \tilde{\Delta}_{s}^{i}\left(\tilde{u}_{s}^{i}+\tilde{K}_{s}^{i} \tilde{x}_{s}^{i}\right) \\
& \left.+\sum_{s=0}^{T-1} \sum_{i \in\{1,2\}}\left(w_{s}^{i}\right)^{\top} \Pi_{t+1}^{i} w_{s}^{i}\right],
\end{aligned}
$$

where $\Delta_{s}=R_{s}+B^{\top} P_{s+1} B$ and $\tilde{\Delta}_{s}^{i}=R_{s}^{i i}+\left(B^{i i}\right)^{\top} \Pi_{s+1}^{i} B^{i i}$, $i \in\{1,2\}$.

PROOF For any strategy profile $\mathbf{g}$, define the expected cost to go from time $t$ onwards as

$$
V_{t}(\mathbf{g})=\mathbb{E}^{\mathbf{g}}\left[\sum_{s=t}^{T-1} c_{s}\left(x_{s}, u_{s}\right)+c_{T}\left(x_{T}\right)\right] .
$$

We claim that

$$
\begin{aligned}
& V_{t}(\mathbf{g})=\mathbb{E}^{\mathbf{g}}\left[\hat{x}_{t}^{\top} P_{t} \hat{x}_{t}+\sum_{i \in\{1,2\}}\left(\tilde{x}_{t}^{i}\right)^{\top} \tilde{P}_{t}^{i} \tilde{x}_{t}^{i}\right. \\
&+\sum_{s=t}^{T-1}\left(\hat{u}_{s}+K_{s} \hat{x}_{s}\right)^{\top} \Delta_{s}\left(\hat{u}_{s}+K_{s} \hat{x}_{s}\right) \\
&+\sum_{s=t}^{T-1} \sum_{i \in\{1,2\}}\left(\tilde{u}_{s}^{i}+\tilde{K}_{s}^{i} \tilde{x}_{s}^{i}\right)^{\top} \tilde{\Delta}_{s}^{i}\left(\tilde{u}_{s}^{i}+\tilde{K}_{s}^{i} \tilde{x}_{s}^{i}\right) \\
&\left.+\sum_{s=t}^{T-1} \sum_{i \in\{1,2\}}\left(w_{s}^{i}\right)^{\top} \Pi_{t+1}^{i} w_{s}^{i}\right] .
\end{aligned}
$$

We prove the claim by backward induction. For $t=T$, Lemma 5 implies that

$$
V_{T}(\mathbf{g})=\mathbb{E}\left[\hat{x}_{T}^{\top} Q_{T} \hat{x}_{T}+\sum_{i \in\{1,2\}}\left(\tilde{x}_{T}^{i}\right)^{\top} Q_{T}^{i i} \tilde{x}_{T}^{i}\right] .
$$

Eq. (29) follows from the definition of $P_{T}$ and $\tilde{P}_{T}^{i}$. This forms the basis of induction. Now assume that (29) is true for $t+1$ and consider $V_{t}$. By definition, we have

$$
V_{t}(\mathbf{g})=\mathbb{E}^{\mathbf{g}}\left[c_{t}\left(x_{t}, u_{t}\right)\right]+V_{t+1}(\mathbf{g})
$$

Using the expression for $V_{t+1}$ and Lemma 9 , we get the expression for $V_{t}$. This completes the induction step and proves the claim (29).

The result of the Theorem then follows from observing that $J(\mathbf{g})=V_{0}(\mathbf{g})$. 


\section{H. Proof of Theorem 1}

By Lemma 3, there is no loss of optimality in restricting attention to control strategy profile of the form (16). By Theorem 2, the performance of a strategy of the form 16) is given by (27). Note that the first two and the last terms of 27) are control free (i.e., they depend on only primitive random variables). Thus, minimizing $J(\mathbf{g})$ is equivalent to minimizing

$$
\begin{aligned}
\tilde{J}(\mathbf{g})=\mathbb{E}^{\mathbf{g}\left[\sum_{s=0}^{T-1}\right.}[ & \left(\hat{u}_{s}+K_{s} \hat{x}_{s}\right)^{\top} \Delta_{s}\left(\hat{u}_{s}+K_{s} \hat{x}_{s}\right) \\
& \left.\left.+\sum_{i \in\{1,2\}}\left(\tilde{u}_{s}^{i}+\tilde{K}_{s}^{i} \tilde{x}_{s}^{i}\right)^{\top} \tilde{\Delta}_{s}^{i}\left(\tilde{u}_{s}^{i}+\tilde{K}_{s}^{i} \tilde{x}_{s}^{i}\right)\right]\right] .
\end{aligned}
$$

By (A3), $R_{t}$ is symmetric and positive definite and therefore so is $R_{t}^{i i}$. It can be shown recursively that $P_{t}$ and $\tilde{P}_{t}$ are symmetric and positive semi-definite. Hence both $\Delta_{t}$ and $\tilde{\Delta}_{t}^{i}$ are symmetric and positive definite. Therefore, $\tilde{J}(\mathbf{g}) \geq 0$ with equality if and only if the strategy profile $\mathrm{g}$ is given by Theorem 1 .

\section{Discussion}

The model in [1] consisted of $N$ local controllers and one remote controller. We restricted our discussion to $N=2$. All steps of our proof apart from Lemma 7 extend trivially to the case of general $N$. To extend Lemma 7 to the case of general $N$, one can establish the following result.

Lemma 10 For the system with general $N$, for any $\gamma=$ $\left(\gamma^{1}, \ldots, \gamma^{n}\right), \gamma^{i} \in\{0,1\}$, we have

$$
\begin{aligned}
\mathbb{E}\left[\hat{x}_{t+1}^{\top} P_{t+1} \hat{x}_{t+1}+\sum_{i=1}^{N}\left(\tilde{x}_{t+1}^{i}\right)^{\top} \tilde{P}_{t+1}^{i} \tilde{x}_{t+1}^{i} \mid \Gamma_{t+1}=\gamma\right] \\
=\mathbb{E}\left[\left(\hat{x}_{t+1}^{\mathrm{OFF}}\right)^{\top} P_{t+1} \hat{x}_{t+1}^{\mathrm{OFF}}\right. \\
\left.\quad+\sum_{i=1}^{N}\left(\tilde{x}_{t+1}^{i, \mathrm{OFF}}\right)^{\top} \Lambda_{t+1}^{i}\left(\gamma^{i}\right) \tilde{x}_{t+1}^{i, \mathrm{OFF}} \mid \Gamma_{t+1}=\gamma\right]
\end{aligned}
$$

where $\Lambda_{t+1}^{i}\left(\gamma^{i}\right)= \begin{cases}\tilde{P}_{t+1}^{i}, & \text { if } \gamma^{i}=0 \\ P_{t+1}^{i i} & \text { if } \gamma^{i}=1 .\end{cases}$

Lemma [7] then follows from observing that

$$
\begin{aligned}
& \sum_{\left(\gamma^{1}, \ldots, \gamma^{N}\right) \in\{0,1\}^{N}} \mathbb{P}\left(\Gamma_{t}^{1}=\gamma^{1}\right) \cdots \mathbb{P}\left(\Gamma_{t}^{N}=\gamma^{N}\right) \Lambda_{t+1}^{i}\left(\gamma^{i}\right) \\
= & \sum_{\gamma^{i} \in\{0,1\}} \mathbb{P}\left(\Gamma_{t}^{i}=\gamma^{i}\right) \Lambda_{t+1}^{i}\left(\gamma^{i}\right) \\
= & p^{i} \tilde{P}_{t+1}^{i}+\left(1-p^{i}\right) P_{t+1}^{i i} \\
= & \Pi_{t+1}^{i} .
\end{aligned}
$$

The proof of Theorem 2 is similar in spirit to the proof of centralized linear quadratic control presented in [3]. However, due to decentralized information and the presence of unreliable communication channels, the specific details are different. As far as we are aware, this is the first paper which presents a methodology to synthesize optimal controllers for dynamic decentralized control systems without using a dynamic programming or a spectral decomposition argument. In contrast to dynamic programming based approaches, we sidestep the subtle measurability issues that arise in common information based dynamic program for continuous state and action spaces. In contrast to spectral decomposition based arguments, we do not apriori restrict attention to linear strategies. We believe that the solution approach presented in this paper is interesting in its own right and may be applicable to other decentralized control problems as well.

\section{ACKNOWLEDGMENT}

The authors are grateful to Ashutosh Nayyar and Yi Ouyang for helpful feedback.

\section{REFERENCES}

[1] S. M. Asghari, Y. Ouyang, and A. Nayyar, "Optimal local and remote controllers with unreliable uplink channels," IEEE Transactions on Automatic Control, 2019 (in print).

[2] A. Nayyar, A. Mahajan, and D. Teneketzis, "Decentralized stochastic control with partial history sharing: A common information approach," IEEE Trans. Autom. Control, vol. 58, no. 7, pp. 1644-1658, July 2013.

[3] K. J. Astrom, Introduction to stochastic control theory. Academic Press New York, 1970.

[4] D. Blackwell, "Memoryless strategies in finite-stage dynamic programming," Ann. Math. Statist., vol. 35, no. 2, pp. 863-865, 061964.

[5] A. Mahajan and S. Tatikonda, "An algorithmic approach to identify irrelevant information in sequential teams," Automatica, vol. 61, pp. 178 $-191,2015$. 